\section{Intravitreal bevacizumab for type 1 idiopathic macular telangiectasia}

\begin{abstract}
Purpose To evaluate the effect of intravitreal bevacizumab injection for treating type 1 idiopathic macular telangiectasia (IMT). Methods Retrospective case series of five eyes of five male patients with type 1 IMT that were treated with 2-3 injections of intravitreal bevacizumab. Best-corrected visual acuity, foveal thickness obtained by optical coherence tomography, and fluorescein angiography (FA) were monitored over a period of up to 12 months.

Results The average follow-up period was 17.0 months (range, 12-21 months). The mean logarithm of the minimal angle of resolution visual acuity was 0.295 at baseline and $0.254(P=0.194)$ and 0.311 $(P=0.461)$ at 3 and 12 months, respectively, after the initial injection. At 12 months, visual acuity had improved in one eye, remained stable in three eyes, and decreased in one eye. The mean foveal thickness was $479 \mu \mathrm{m}$ at baseline; at 1 month after the therapy, marked reduction of macular oedema was seen only in one eye. The mean foveal thickness was $418 \mu \mathrm{m}(P=0.287)$ and $473 \mu \mathrm{m}$ $(P=0.482)$ at 3 and 12 months after the initial injection, respectively. At 12 months, the foveal thickness had decreased by $>100 \mu \mathrm{m}$ in one eye, but had increased by $>100 \mu \mathrm{m}$ in two eyes. FA did not show a reduction in late leakage.

Conclusions Treatment with intravitreal bevacizumab does not appear to improve visual acuity or retinal oedema in type 1
\end{abstract} IMT.

Eye (2010) 24, 1492-1497; doi:10.1038/eye.2010.61; published online 30 April 2010

Keywords: bevacizumab; idiopathic juxtafoveolar retinal telangiectasis; idiopathic macular telangiectasia; vascular endothelial growth factor
K Takayama, S Ooto, H Tamura, K Yamashiro, A Otani, A Tsujikawa and N Yoshimura

Introduction

Idiopathic macular telangiectasia (IMT) is characterized by vascular anomalies affecting the macular capillary network. Idiopathic juxtafoveolar retinal telangiectasis (IJRT) was first proposed in 1982 by Gass and Oyakawa; ${ }^{1}$ it was further classified by Gass and Blodi in 1993. ${ }^{2}$ Yannuzzi et al ${ }^{3}$ recently classified it as type 1 (aneurysmal telangiectasia) and type 2 (parafoveal telangiectasia).

Type 1 IMT or unilateral parafoveal telangiectasis (Group 1B IJRT) typically affects men (mean age of onset, 40 years). The telangiectasis is generally confined unilaterally to the temporal half of the macula in an area of 1-2 disc diameters, with equivalent areas superior and inferior to the horizontal raphe involved. Visual loss is mainly caused by macular oedema and exudation.

There is no established treatment for type 1 IMT, although some type 1 IMT patients responded to laser photocoagulation or photodynamic therapy (PDT). ${ }^{4,5}$ Recently, Gamulescu et $a l^{6}$ reported that a single intravitreal bevacizumab injection markedly increased visual acuity in a type 1 IMT patient, suggesting that anti-vascular endothelial growth factor (VEGF) therapy may be effective in treating the associated visual deterioration. As this was reported in only one patient, the efficacy of this therapy is controversial.

In this study, we used bevacizumab to treat type 1 IMT.

\section{Materials and methods}

Type 1 IMT patients treated with bevacizumab were retrospectively reviewed at the Macular Service Department, Kyoto University Hospital, Kyoto, Japan. Five eyes of five male patients (mean age, 69.8 years (range, 57-80 years)) were naive to treatment. After the off-label uses of bevacizumab and its potential risks and benefits 
were explained, written informed consent was obtained. This study was approved by the institutional review board and the ethics committee at Kyoto University Graduate School of Medicine, and it adhered to the tenets of the Declaration of Helsinki.

All patients underwent a comprehensive ophthalmologic examination, including measurements of best-corrected visual acuity (BCVA) and intraocular pressure, indirect ophthalmoscopy, slit-lamp biomicroscopy with a contact lens, colour fundus photography, simultaneous fluorescein angiography (FA), and indocyanine angiography (IA), by using a confocal laser scanning ophthalmoscope (HRA2; Heidelberg Engineering, Dossenheim, Germany) and optical coherence tomography (OCT).

Diagnosis of type 1 IMT was based on the fundus examination, FA, IA, and OCT after exclusion of neovascular maculopathy (ie age-related macular degeneration, polypoidal choroidal vasculopathy, retinal angiomatous proliferation, pathologic myopia, idiopathic choroidal neovascularization, and angioid streaks), other causes of secondary macular telangiectasia (ie Coat's disease, Leber's disease, retinal vein occlusion, and radiation retinopathy), and diabetes. At the initial visit, all eyes had cystoid macular oedema (CME) and showed prompt filling of telangiectatic vessels, affecting both the superficial and deep capillary network, as well as late intraretinal staining on FA.

Treatment was administered on an outpatient basis and performed under sterile conditions. The eye was prepared with povidone iodine $5 \%$. The eyelids were stabilized with an eyelid speculum, and $1.25 \mathrm{mg}(0.05 \mathrm{ml})$ bevacizumab was intravitreally administered $3.5 \mathrm{~mm}$ posterior to the limbus through the pars plana with a 30-gauge needle under topical anaesthesia. Intraocular pressure was then measured. Prophylactic topical antibiotics were applied for the following 1 week. All patients were examined for changes in BCVA or retinal thickness 2 weeks after treatment; if macular oedema did not reduce, additional treatments were performed 1-2 times at the discretion of the physician at 4-week intervals. If macular oedema reduced, patients were examined monthly and re-treated if OCT showed a $>100-\mu \mathrm{m}$ increase in macular thickness.
OCT was performed at each visit. Serial SD-OCT B-scan images were obtained with ophthalmoscope C7 (Nidek, Gamagori, Japan), Cirrus HD-OCT (Carl Zeiss Meditec, Dublin, CA, USA), 3D-OCT-1000 (Topcon, Tokyo, Japan), or Spectralis HRA + OCT (Heidelberg Engineering, Dossenheim, Germany). To evaluate foveal thickness before and after treatment, we chose 2-5 B-scan images from the vertical and horizontal line scans across the fovea and radial scans centred at the fovea. Foveal thickness - distance between the inner limiting membrane and inner border of the retinal pigment epithelium at the foveal centre-was then measured with the computer-based caliper measurement tool built into the OCT system. Foveal thickness was defined as the mean thickness from the B-scan images.

All values are presented as mean (SD). Visual acuity was converted to logarithm of the minimal angle of resolution (logMAR). Foveal thickness and logMAR before and after treatment were compared using the paired $t$-test. $P<0.05$ was considered significant. Statistical analysis was conducted using SPSS17 (SPSS Inc., Chicago, IL, USA).

\section{Results}

The average follow-up period was 16.0 (range, 12-26) months. At baseline, all eyes showed prominent parafoveal capillaries, microaneurysms, and CME. For all patients, FA showed late leakage, and OCT revealed foveal intraretinal hyporeflective spaces, suggesting CME. The number of treatments administered was two for four eyes and three for one eye.

The mean logMAR was 0.295 (Table 1), 0.254 $(P=0.194)$, and $0.311(P=0.461)$ at the baseline and 3 and 12 months after initial treatment, respectively. At 3 months, visual acuity was stable in all eyes; however, at 12 months, visual acuity improved in one eye, was stable in three eyes, and decreased in one eye (Table 2). In Patient C, CME persisted, but the foveal lipids had disappeared; this might have resulted in an improved visual acuity. In Patient E, visual acuity decreased in accordance with the worsened retinal oedema. At the last follow-up, the mean $\log$ MAR was $0.464(P=0.170)$.

Table 1 Mean logMAR visual acuity of eyes with type 1 idiopathic macular terangiectasia at several time points after intravitreal bevacizumab

\begin{tabular}{|c|c|c|c|c|c|c|c|}
\hline & Baseline & $1 M$ & $3 M$ & $6 M$ & $9 M$ & $12 M$ & Last follow-up \\
\hline Mean $\log$ MAR visual acuity & 0.295 & 0.254 & 0.254 & 0.199 & 0.314 & 0.311 & 0.464 \\
\hline$P$-value & & 0.194 & 0.194 & 0.132 & 0.443 & 0.461 & 0.170 \\
\hline Mean foveal thickness $(\mu \mathrm{m})$ & 479 & 408 & 418 & 500 & 472 & 473 & 460 \\
\hline$P$-value & & 0.213 & 0.287 & 0.349 & 0.480 & 0.482 & 0.423 \\
\hline
\end{tabular}




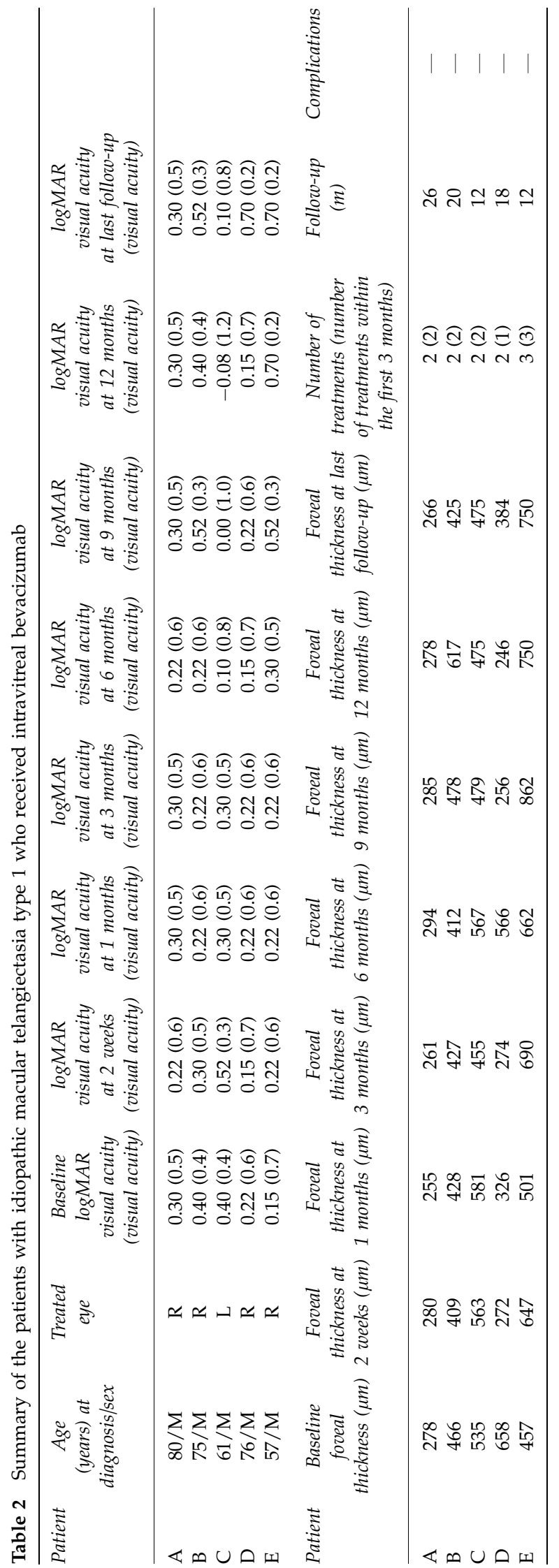

The mean baseline foveal thickness was $479 \mu \mathrm{m}$ (Table 1). One month after treatment, marked reduction in retinal oedema was observed only in one eye. The mean foveal thickness was $418 \mu \mathrm{m}(P=0.287)$ and $473 \mu \mathrm{m}$ $(P=0.482)$ at 3 and 12 months after initial treatment, respectively. At 12 months, the foveal thickness had decreased by $>100 \mu \mathrm{m}$ in one eye, but had increased by $>100 \mu \mathrm{m}$ in two eyes. At the last follow-up, it was $460 \mu \mathrm{m}$ $(P=0.423)$. Intraretinal cystic spaces persisted in all eyes, although some decreased in size.

FA was performed after bevacizumab treatment in two eyes (Patients B and C); however, there was no reduction in late leakage.

BCVA and foveal thickness before and after treatment are summarized in Table 2.

\section{Selected case report}

Patient E: A 57-year-old man had visual loss and metamorphopsia in his right eye for several months. Fundus examination and OCT showed microaneurysms and CME in the eye (Figure 1). BCVA in this eye was 0.7. FA showed prominent parafoveal capillaries, microaneurysms in the early phase, and leakage in the late phase (Figure 1). OCT showed intraretinal cystoid spaces; foveal thickness was $457 \mu \mathrm{m}$ (Figure 2). No abnormal findings were noted in his left eye.

Bevacizumab was administered intravitreally three times at 4-week intervals. Three months after the first injection, BCVA was 0.6, and foveal thickness, $690 \mu \mathrm{m}$ (Figure 2); at 6 months, they were 0.5 and $662 \mu \mathrm{m}$, respectively (Figure 2); at 12 months, 0.2 and $750 \mu \mathrm{m}$, respectively (Figure 2). Visual acuity and foveal thickness worsened in this patient despite intravitreal bevacizumab injections for 3 months.

\section{Discussion}

To date, there is no established treatment protocol for type 1 and type 2 IMT. However, numerous methods have been used for the treatment of type 2 IMT, including photocoagulation, ${ }^{7}$ surgical removal of the subretinal neovascular membrane, ${ }^{8}$ transpupillary thermotherapy, ${ }^{9,10} \mathrm{PDT}_{1}^{11,12}$ intravitreal triamcinolone acetonide administration, ${ }^{13-15}$ and intravitreal bevacizumab administration. ${ }^{6,16-21}$ The results of these studies showed that bevacizumab was somewhat efficacious for the treatment of type 2 IMT.

In contrast, there are few reports on the treatment of type 1 IMT. Photocoagulation, ${ }^{4} \mathrm{PDT}^{5}$ and intravitreal bevacizumab ${ }^{6}$ have been reported to benefit type 1 IMT patients; however, the sample sizes were small. Chopder et $a l^{4}$ reported the use of photocoagulation for three cases of type 1 IMT (initial visual acuity: 6/36, 6/24, and 6/12; 


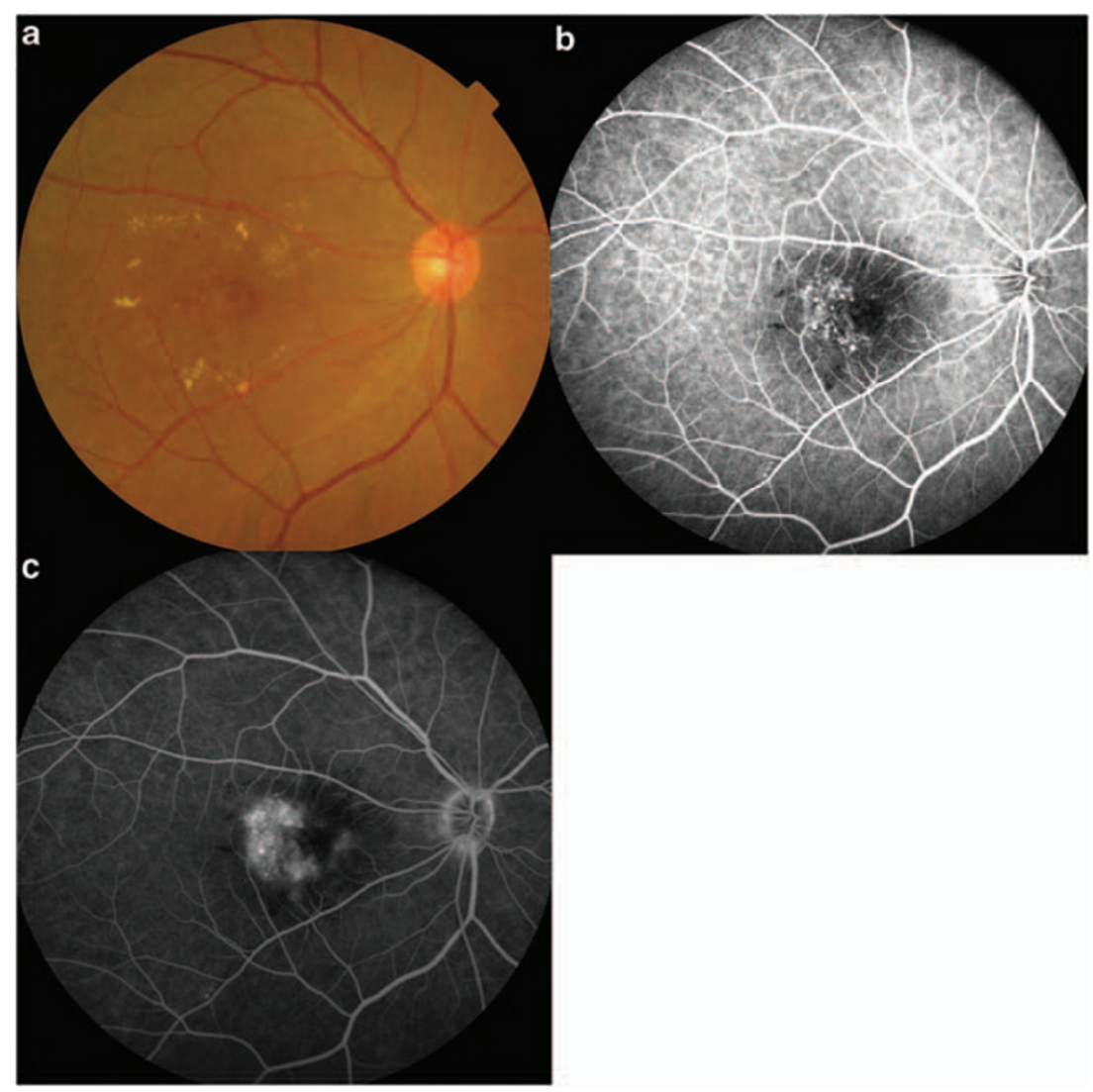

Figure 1 Patient E: A 57-year-old man presented with a history of several months of visual loss and metamorphopsia in his right eye. (a) Fundus examination showed microaneurysms and cystoid macular oedema in his right eye. Best-corrected visual acuity (BCVA) in his right eye was 0.7. (b, c) Fluorescein angiography (FA) showed prominent parafoveal capillaries and microaneurysms in the early phase (b) and leakage in the late phase (c).

eventual post-treatment visual acuity: $6 / 12,6 / 12$, and $6 / 12$, respectively). The effectiveness of PDT was reported in one case of type 1 IMT (initial visual acuity, 20/200; 3 months after PDT, 20/40). ${ }^{5}$ Visual acuity remained stable up to 32 months after treatment. Gamulescu $e t \mathrm{al}^{6}$ recently reported that a single injection of intravitreal bevacizumab markedly improved visual acuity in a type 1 IMT patient.

We followed up five type 1 IMT patients treated for $>12$ weeks with bevacizumab. The mean visual acuity and mean foveal thickness before and after intravitreal bevacizumab administration were not considerably different. At the last follow-up, all eyes had CME, and FA showed late leakage. Therefore, the effect of bevacizumab seems to be limited in type 1 IMT patients compared with type 2 IMT patients.

VEGF has an integral part in the formation of abnormal blood vessels and in increasing vascular permeability in many pathological conditions. Increased vascular permeability and SRNV are observed in type 2 IMT; therefore, VEGF may have an essential function in the pathogenesis of type 2 IMT. Green $e t a l^{22}$ described thickening of the wall of the retinal capillaries resulting from proliferation of the basement membrane and narrowing of the capillary lumen in a type 2 IMT eye. Degeneration of pericytes and endothelial cells was also observed. They hypothesized that these structural capillary changes could disturb oxygen metabolism between the lumen and neurosensory retina, resulting in hypoxia-induced VEGF release.

It is unknown whether VEGF has a function in the pathogenesis of type 1 IMT. Neovascularization has not been reported in type 1 IMT, in contrast to type 2 IMT. This study showed that the effect of bevacizumab appears to be limited in type 1 IMT compared with type 2 IMT. Intraretinal cystic spaces persisted in all eyes even after intravitreal bevacizumab administration. Thus, it is possible that the pathophysiology, including the involvement of VEGF, in the 2 types of IMT may be considerably different.

Intravitreal bevacizumab treatment may rapidly improve retinal oedema in other diseases. Prager $e t a l^{23}$ 

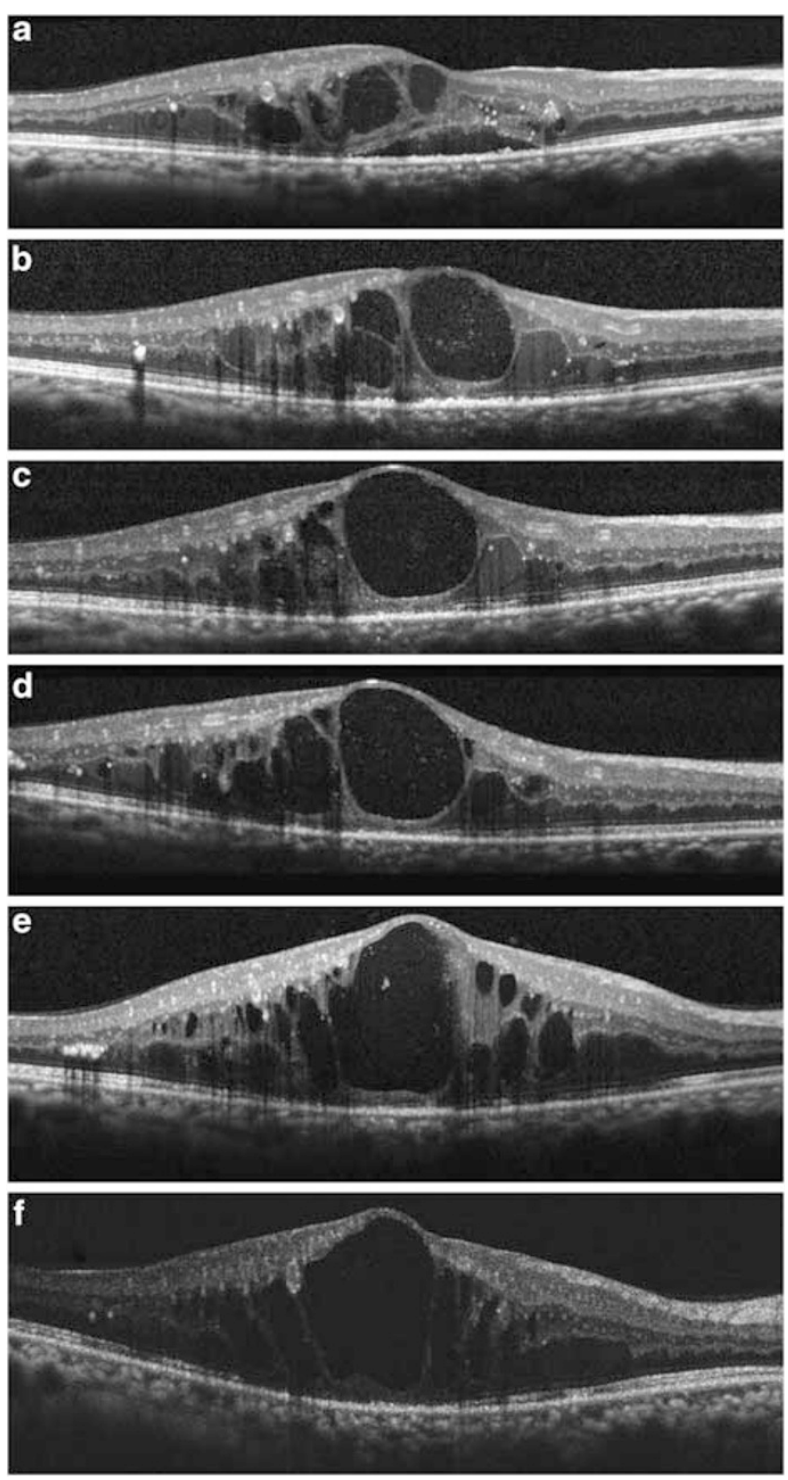

Figure 2 Optical coherence tomography (OCT) images of Patient E. (a) At baseline, OCT showed intraretinal cystoid spaces and serous retinal detachment (SRD), and the foveal thickness was $457 \mu \mathrm{m}$. Best-corrected visual acuity (BCVA) was 0.7. Intravitreal injection of bevacizumab was administered three times at intervals of 4 weeks each. (b) At 1 month after intravitreal bevacizumab, BCVA was 0.6 and the foveal thickness was $501 \mu \mathrm{m}$. SRD was resolved; however, the intraretinal cystoid spaces were increased. (c) At 3 months, BCVA was 0.6 and the foveal thickness was $690 \mu \mathrm{m}$. (d) At 6 months, BCVA was 0.5 and the foveal thickness was $662 \mu \mathrm{m}$. (e) At 9 months, BCVA was 0.3 and the foveal thickness was $862 \mu \mathrm{m}$. (f) At 12 months, BCVA was 0.2 and the foveal thickness was $750 \mu \mathrm{m}$. The visual acuity and retinal oedema had worsened despite three intravitreal injections of bevacizumab.

reported that retinal thickness decreased rapidly, even at 1 day, after intravitreal bevacizumab injection in eyes with macular oedema secondary to retinal vein occlusion. Therefore, all our patients were examined for changes in BCVA or retinal thickness 2 weeks after treatment. However, we found a marked reduction in retinal thickness only in one eye; most eyes did not respond. In Patient E, retinal oedema worsened despite monthly bevacizumab injections. Therefore, we consider that many eyes with type 1 IMT show little response to intravitreous bevacizumab.

Thus, despite the limitations of this study - its retrospective nature, small sample size, lack of controls, and lack of protocol refractions - we think that intravitreal bevacizumab does not increase visual acuity or reduce macular oedema in most type 1 IMT patients. Therefore, we do not advocate its use for type 1 IMT.

\section{Summary \\ What was known before \\ - To date, there are few reports concerning the treatment of type 1 idiopathic macular telangiectasia (IMT) and only one case report has shown the efficacy of anti-VEGF therapy. \\ What this study adds \\ - We could follow five cases of type 1 IMT treated for $>12$ weeks by intravitreal bevacizumab and found that treatment with intravitreal bevacizumab does not appear to improve visual acuity or retinal oedema in type1 IMT.}

\section{Conflict of interest}

The authors declare no conflict of interest.

\section{Acknowledgements}

This research was supported in part by a Grant-in-Aid for Scientific Research (21791679) from the Japan Society for the Promotion of Science (JSPS).

\section{References}

1 Gass JD, Oyakawa RT. Idiopathic juxtafoveolar retinal telangiectasis. Arch Ophthalmol 1982; 100: 769-780.

2 Gass JDM, Blodi BA. Idiopathic juxtafoveolar retinal telangiectasis: update of classification and follow-up study. Ophthalmology 1993; 100: 1536-1546.

3 Yannuzzi LA, Bardal AM, Freund KB Chen KJ, Eandi CM, Blodi B. Idiopathic macular telangiectasia. Arch Ophthalmol 2006; 124: 450-460.

4 Chopder A. Retinal telangiectasis in adults: fluorescein angiographic findings and treatment by argon laser. $\mathrm{Br} J$ Ophthalmol 1978; 62: 243-250.

5 Kotoula MG, Chatzoulis DZ, Karabatsas CH, Tsiloulis A, Tsironi EE. Resolution of macular edema in idiopathic juxtafoveal telangiectasis using PDT. Ophthalmic Surg Lasers Imaging 2009; 40: 65-67. 
6 Gamulescu MA, Walter A, Sachs H, Helbig H. Bevacizumab in the treatment of idiopathic macular telangiectasia. Graefes Arch Clin Ophthalmol 2008; 246: 1189-1193.

7 Park DW, Schatz H, McDonald HR, Johnson RN. Grid laser photocoagulation for macular edema in bilateral juxtafoveal telangiectasis. Ophthalmology 1997; 104: 1838-1846.

8 Berger AS, McCuen 2nd BW, Brown GC, Brownlow RL Jr. Surgical removal of subfoveal neovascularization in idiopathic juxtafoveolar retinal telangiectasis. Retina 1997; 17: 94-98.

9 Shukla D, Singh J, Kolluru CM, Kim R, Namperumalsamy P. Transpupillary thermotherapy for subfoveal neovascularization secondary to group $2 \mathrm{~A}$ idiopathic juxtafoveolar telangiectasis. Am J Ophthalmol 2004; 138: 147-149.

10 Nachiappan K, Shanmugam MP. Treatment of CNVM secondary to idiopathic juxtafoveal retinal telangiectasis by transpupillary thermotherapy. Am J Ophthalmol 2005; 139: 577-578.

11 Snyers B, Verougstraete C, Postelmans L, Leys A, Hykin P. Photodynamic therapy of subfoveal neovascular membrane in type 2A idiopathic juxtafoveolar retinal telangiectasis. Am J Ophthalmol 2004; 137: 812-819.

12 Potter MJ, Szabo SM, Sarraf D, Michels R, Schmidt-Erfurth U. Photodynamic therapy for subretinal neovascularization in type 2A idiopathic juxtafoveolar telangiectasis. Can J Ophthalmol 2006; 41: 34-37.

13 Alldredge CD, Garretson BR. Intravitreal triamcinolone for the treatment of idiopathic juxtafoveal telangiectasis. Retina 2003; 23: 113-116.

$14 \mathrm{Wu}$ L, Evans T, Arevalo JF, Berrocal MH, Rodriguez FJ, Hsu $\mathrm{M}$ et al. Long-term effect of intravitreal triamcinolone in the nonproliferative stage of type II idiopathic parafoveal telangiectasia. Retina 2008; 28: 314-319.

15 Smithen LM, Spaide RF. Photodynamic therapy and intravitreal triamcinolone for a subretinal neovascularization in bilateral idiopathic juxtafoveal telangiectasis. Am J Ophthalmol 2004; 138: 884-885.

16 Maia Jr OO, Bonanomi MT, Takahashi WY, Nascimento VP, Takahashi BS. Intravitreal bevacizumab for foveal detachment in idiopathic perifoveal telangiectasia. Am J Ophthalmol 2007; 114: 296-299.

17 Kovach JL, Rosenfeld PJ. Bevacizumab (avastin) therapy for idiopathic macular telangiectasia type II. Retina 2009; 29: 27-32.

18 Charbel Issa P, Holz FG, Scholl HPN. Findings in fluorescein angiography and optical coherence tomography after intravitreal bevacizumab in type 2 idiopathic macular telangiectasia. Ophthalmology 2007; 114: 1736-1742.

19 Charbel Issa P, Finger RP, Holz FG, Scholl HPN. Eighteenmonth follow-up of intravitreal bevacizumab in type 2 idiopathic macular telangiectasia. Br J Ophthalmol 2008; 92: 941-945.

20 Jorge R, Costa RA, Calucci D, Scott IU. Intravitreal bevacizumab (Avastin) associated with the regression of subretinal neovascularization in idiopathic juxtafoveolar retinal telangiectasis. Graefes Arch Clin Exp Ophthalmol 2007; 245: 1045-1048.

21 Rishi P, Rishi E, Shroff D. Combined photodynamic therapy and intravitreal bevacizumab as primary treatment for subretinal neovascularization associated with type 2 idiopathic macular telangiectasia. Indian J Ophthalmol 2009; 57: 241-242.

22 Green WR, Quigley HA, de la Cruz Z, Cohen B. Parafoveal retinal telangiectasis: light and electron microscopy studies. Trans Ophthalmol Soc UK 1980; 100: 162-170.

23 Prager F, Michels S, Kriechbaum K, Georgopoulos M, Funk M, Geitzenauer W et al. Intravitreal bevacizumab (Avastin) for macular oedema secondary to retinal vein occlusion: 12-month results of a prospective clinical trial. Br J Ophthalmol 2009; 93: 452-456. 